Trauma Berufskrankh 2008 · 10[Suppl 1]:78-80 DOI 10.1007/s10039-007-1261-0

Online publiziert: 15. Dezember 2007

๑) Springer Medizin Verlag 2007

\author{
J. Breuer \\ Deutsche Gesetzliche Unfallversicherung, Sankt Augustin
}

\title{
Wandel der gesetzlichen Unfallversicherung
}

Die gesetzliche Unfallversicherung im Wandel - nie war diese Feststellung berechtigter als zum jetzigen Zeitpunkt. Deswegen würde es den hier zur Verfügung stehenden Raum sprengen, unternähme man den Versuch, alle Aspekte dieses Wandels umfassend darzulegen. Ich möchte daher in der gebotenen Kürze nur einige ausgewählte „Reformbaustellen“ benennen und anschließend einige aktuelle Herausforderungen für die Heilverfahren der Unfallversicherung aufzeigen.

\section{Organisationsreform}

Nachdem im politischen Raum bereits Forderungen erhoben wurden, die gewerbliche Unfallversicherung als Einheitsträger zu organisieren oder aber nur noch einige wenige Berufsgenossenschaften zu belassen, haben die Selbstverwaltungen der Träger und der Verbände das Heft des Handelns selbst in die Hand genommen. Dadurch ist es gelungen, ein zukunftsfähiges Konzept vorzulegen. Es sieht eine Reduzierung der Trägerzahl vor, ohne dabei das Branchenprinzip aufzugeben. In absehbarer Zeit wird es danach nur noch 9 gewerbliche Berufsgenossenschaften geben. Dieser Ansatz hat inzwischen auch beim Gesetzgeber Eindruck gemacht. Eine weitergehende Reduzierung der Trägerzahl ist daher nicht mehr zu erwarten.

Offen ist demgegenüber noch die Frage nach der Ausgestaltung des zukünftig einheitlichen Spitzenverbands der öffentlichen und der gewerblichen Unfallversicherung. Während die Unfallversicherung für eine Fortführung der schon bislang erfolgreich und reibungslos praktizierten Vereinslösung eintritt, streben der Bund und eine Reihe von Ländern die Verkörperschaftung des Spitzenverbands und damit die Schaffung einer neuen Be- hörde an. Wir hoffen aber, dass wir die politischen Entscheidungsträger letztlich von den Vorteilen der von uns entwickelten Vereinslösung für den gemeinsamen Dachverband überzeugen können.

Neben der organisationsrechtlichen Neuordnung der Unfallversicherung ist nach heutigem Stand auch eine verstärkte Konturierung der Trägerzuständigkeiten zu erwarten. Dies soll insbesondere durch eine ergänzende gesetzliche Regelung und die Einrichtung einer internen Schiedsstelle gewährleistet werden. Natürlich tragen auch die angesprochenen $\mathrm{Fu}-$ sionen artverwandter Träger zum Abbau von Zuständigkeitsproblemen bei.

Die Folgen des wirtschaftlichen Strukturwandels sollen zukünftig durch ein neues Konzept einer solidarischen Verteilung der so genannten Überaltlasten bewältigt werden.

Jeder dieser Punkte verdiente eigentlich eine ausführlichere Würdigung. Da hierfür aus verständlichen Gründen kein Raum ist, soll es bei diesem Hinweis bleiben. All diese, vom Gesetzgeber in weiten Teilen aufgenommenen Vorschläge der gewerblichen Berufsgenossenschaften machen eines deutlich: Selbstverwaltung ist keine bloße Floskel, sondern ein wirksames Instrument, um notwendige Änderungen im Interesse aller Betroffenen, Arbeitnehmern wie Arbeitgebern, zu gestalten.

\section{Reform des Rentenrechts}

Im Bereich des Entschädigungsrechts hat sich das Fachministerium seit mehr als 9 Monaten um die Umsetzung des Eckpunktepapiers bemüht, das die Bund-LänderArbeitsgruppe im Juni 2006 zur Reform der gesetzlichen Unfallversicherung erarbeitet hat. Ein zusammenhängendes Konzept wird erst in diesen Tagen vorgelegt.
Die bisherige abstrakte Schadensbemessung soll danach durch ein aus 2 Säulen bestehendes Rentenmodell abgelöst werden. Ausgeglichen werden soll einerseits ein (mehr oder weniger) konkret berechneter Erwerbsschaden. Unabhängig hiervon ist ein abstrakter Ausgleich des Gesundheitsschadens vorgesehen. Dieser soll im Wesentlichen nach den Kriterien des sozialen Entschädigungsrechts erfolgen.

Für die im Auftrag der gesetzlichen Unfallversicherung tätigen Gutachter folgt daraus: Die Kriterien des sozialen Entschädigungsrechts einerseits und die für die Bewertung von Arbeitsunfällen sowie Berufskrankheiten maßgeblichen Maßstäbe andererseits werden sich aller Voraussicht nach vermischen. Noch ist es zu früh, diese Entwicklung abschließend zu bewerten. Wenn die vom Ministerium momentan vorgeschlagene Lösung umgesetzt wird, kommt auf die Unfallversicherungsträger sowie die Gutachter eine Zeit intensiver Anstrengungen zu, um das neue Recht für die praktische Arbeit aufzubereiten.

\section{Bestätigung der Heilverfahren}

Im Gegensatz zum Rentenrecht gehen wir für den Bereich der Rehabilitation und Heilbehandlung nach dem derzeitigen Stand nicht davon aus, dass die Reform wesentliche Änderungen bringen wird. Die Eckpunkte der Bund-LänderArbeitsgruppe haben den hohen qualitativen Stand der Heilbehandlung in der gesetzlichen Unfallversicherung vielmehr ausdrücklich gewürdigt und eine optimale Versorgung attestiert.

Angesichts der immer kürzer werdenden Halbwertszeiten gesundheitspolitischer Reformen ist diese positive Hervorhebung seitens der Politik durchaus bemerkenswert. Sie zeigt einmal mehr die 
hohe Akzeptanz, die die gesetzliche Unfallversicherung, aber auch die mit ihr zusammenwirkenden Leistungserbringer in Gesellschaft und Politik erfahren.

\section{Verbesserung von Effektivität und Effizienz}

So sehr wir uns über diese Bestätigung freuen, folgt daraus nicht, dass wir uns mit dem bisher erreichten Niveau zufrieden geben und keine weiteren Anstrengungen zur Optimierung unserer Heilverfahren unternehmen. Aus der besonderen Verpflichtung, eine optimale Heilbehandlung mit allen geeigneten Mitteln sicherzustellen und die Verunfallten bestmöglich wieder in Beruf und Gesellschaft einzugliedern, ergibt sich für die gesetzliche Unfallversicherung die fortwährende Aufgabe, die Effektivität, aber auch die Effizienz ihrer Leistungen zu hinterfragen und auf Verbesserungen hinzuwirken.

Dabei dürfen wir nicht dazu übergehen, die Effizienz unserer Heilverfahren - also das Verhältnis von Aufwand und Nutzen - ausschließlich mit reinen Kostendämpfungsmaßnahmen zu verbessern. So wichtig die Verhältnismäßigkeit des Mitteleinsatzes auch ist: Eine „BilligReha" entspricht weder dem gesetzlichen Auftrag noch dem Selbstverständnis einer sozialen und selbst verwalteten gesetzlichen Unfallversicherung! Denn wirtschaftliches Handeln hat sich in der Unfallversicherung nicht allein an den für die Rehabilitation entstehenden direkten Kosten zu orientieren. Es ist vielmehr das gesamte Spektrum von der Akutbehandlung über die Rehabilitation bis hin zur Rentenzahlung zu berücksichtigen. Daraus folgt: Je hochwertiger und erfolgreicher die Heilbehandlung und Rehabilitation, desto weniger Entschädigungsleistungen sind am anderen Ende der Kette erforderlich. Der Grundsatz „Reha vor Rente“ hat für uns deshalb nicht nur eine sozialpolitische Dimension, sondern auch eine große wirtschaftliche Bedeutung.

\section{Qualitätssicherung}

Eine Lockerung der Anforderungen für die Beteiligung von Leistungserbringern an den besonderen Heilverfahren, die mit einer Gefährdung der Behandlungsergeb-

Trauma Berufskrankh 2008 · 10[Suppl 1]:78-80 DOI 10.1007/s10039-007-1261-0

(c) Springer Medizin Verlag 2007

\section{J. Breuer \\ Wandel der gesetzlichen Unfallversicherung}

\section{Zusammenfassung}

Die gesetzliche Unfallversicherung befindet sich derzeit in einem Umbauprozess, von dem im vorliegenden Beitrag nur einige wichtige Teilgebiete der Organisationsreform angesprochen werden. Zudem werden einige aktuelle Herausforderungen für die Heilverfahren der Unfallversicherung aufgezeigt, wie die Reform des Rentenrechts oder die Neuausrichtung des Verletzungsartenverfahrens. Aber auch die Bestätigung der Heilverfahren, so erfreulich sie ist, bedeutet nicht, dass nur deren Qualität zu sichern ist, sondern auch die Effektivität und Effizienz dieser Verfahren sind ständig zu optimieren. Um den tief greifenden Veränderungen gerecht zu werden, werden die Denkschriften überarbeitet werden müssen, die Qualität und Auswirkungen der bereits durchgeführten und noch zu erfolgenden Veränderung müssen beobachtet und optimiert werden. Ziel ist nach wie vor eine möglichst hohe Behandlungsqualität zu ökonomisch vertretbaren Kosten zu erreichen und nach außen zu dokumentieren, d. h. der Grundatz ${ }_{\text {,Reha }}$ vor Rente" ist nach wie vor gültig.

\section{Schlüsselwörter}

Gesetzliche Unfallversicherung · Organisationsreform · Heilverfahren · Verletzungsartenverfahren · Behandlungsqualität

\section{Changes in legally required accident insurance}

\section{Abstract}

Legally required accident insurance is currently in the process of restructuring, but in this article only a few important subsectors of the organisational reform are addressed. In addition, a few current challenges posed to the treatments covered by accident insurance are highlighted, such as the reform of pension law or the new organisation of the way types of injury are classified. But even the recognition of the treatments, gratifying as it is, does not mean that only their quality has to be assured; work must be ongoing to ensure that the efficacy and efficiency of these procedures are optimum. In order to get to grips with the thorough-going changes, the memoranda must be revised, and the quality and ramifications of changes already made and still to come must be observed and optimised. The object is still to achieve the highest possible quality of treatment at economically justifiable cost and to document it to those outside; this means the principle of rehabilitation rather than pension is still valid.

\section{Keywords}

Legally required accident insurance $\cdot$ Organisational reform - Treatment - Classification of types of injury - Quality of treatment 
nisse verbunden wäre, werden wir auch in Zukunft nicht akzeptieren. Deshalb dürfen nur Ärzte mit besonderer unfallchirurgischer Qualifikation sowie hoch qualifizierte stationäre Einrichtungen die bei uns versicherten Unfallverletzten behandeln. Dies haben wir zuletzt im Zusammenhang mit der Einführung des neuen gemeinsamen Facharztes für Orthopädie und Unfallchirurgie im Jahr 2003 unterstrichen, als wir uns mit Erfolg für die Schaffung einer besonderen unfallchirurgischen Zusatzqualifikation „Spezielle Unfallchirurgie" eingesetzt und diese letztlich umfassend in die Anforderungen zur Beteiligung am D-Arzt- und Verletzungsartenverfahren eingebunden haben.

Jetzt gilt es, die Auswirkungen der neuen Musterweiterbildungsordnung auf die Versorgungslandschaft weiter zu verfolgen. Derzeit besteht zwar noch kein erkennbarer Mangel an D-Ärzten, doch werden wir die weitere Entwicklung genauestens beobachten und bei einer drohenden Unterversorgung in ländlichen oder strukturschwachen Regionen steuernd eingreifen.

\section{Neuausrichtung des Verletzungsartenverfahrens}

Für den stationären Bereich hat die Deutsche Gesellschaft für Unfallchirurgie mit der Veröffentlichung des Weißbuchs zur Schwerverletztenversorgung im vergangenen Jahr richtungweisende Maßstäbe gesetzt.

Die Grundstruktur des Weißbuchs findet sich bereits jetzt im Wesentlichen in unseren Anforderungen für eine Beteiligung am Verletzungsartenverfahren wieder. Die Unfallversicherung ist sich bewusst, dass die Umsetzung der Vorgaben des Weißbuchs ganz wesentlich auch von ihrem Engagement abhängen wird. Es ist daher zu prüfen, ob und welche der im Weißbuch beschriebenen Qualitätssicherungsinstrumente und prozessualen Verbesserungen zusätzlich in unsere Anforderungen aufgenommen werden sollten und welchen Beitrag wir zur Unterstützung des Aufbaus von regionalen Netzwerkstrukturen leisten können.

All diese Fragen sind einzubetten in eine Gesamtdiskussion um die künftige Neuausrichtung des Verletzungsartenver- fahrens. Wir werden intensiv darüber beraten müssen, welche Auswirkungen der tief greifende Wandel des Krankenhaussektors mit seinem klar erkennbaren Trend zur Konzentration und Spezialisierung auf das heutige $\$ 6$-Verfahren mit seiner generellen Zulassung von Krankenhäusern zur Behandlung Unfallverletzter haben wird. Im Angesicht einer sich wandelnden Bedarfslage und der großen Bedeutung schwerer und komplexer Verletzungsfälle deutet vieles darauf hin, dass auch die Unfallversicherungsträger in Zukunft stärker als bisher die in einzelnen Kliniken vorhandene spezialisierte Kompetenz für bestimmte Behandlungsfälle, Operationen oder Versorgungsformen nutzen werden.

Klar ist aber auch, dass weiterhin eine ausreichende Anzahl hoch qualifizierter Traumazentren zur Verfügung stehen muss, in denen eine umfassende und interdisziplinäre Behandlung von Schwer- und Komplexverletzten gewährleistet wird. Das Weißbuch der DGU und die dort aufgezeigte 3-stufige Organisation mit Kliniken unterschiedlicher Ausstattung zeichnet hier den Weg vor. Mit Blick auf die in den Einrichtungen der höchsten Versorgungsstufe vorgehaltenen Sach- und Personalressourcen stehen wir vor der großen Aufgabe, diese Kliniken auch in Zeiten von DRG-Fallpauschalen mit angemessenen Mitteln auszustatten.

\section{Überarbeitung der Denkschriften}

Einer weiteren Verbesserung der Versorgung Schwer- und Komplexverletzter dienen die Überarbeitung der Denkschriften für die Behandlung Querschnittgelähmter, Brandverletzter und Schädel-HirnVerletzter sowie die Neuerstellung von Denkschriften für die Behandlung von Polytraumen und chronischer Osteitis bzw. MRSA. Die Neufassungen der Denkschriften werden derzeit im Auftrag der gesetzlichen Unfallversicherung unter Federführung der Vereinigung Berufsgenossenschaftlicher Kliniken vorbereitet.

\section{Ergebnisqualität im Fokus}

Über die in der gesetzlichen Unfallversicherung traditionell stark ausgeprägten Aspekte der Struktur- und Prozessqualität hinaus wird es in Zukunft mehr noch als bisher darauf ankommen, auch die Ergebnisqualität der Rehabilitation Unfallverletzter ins Blickfeld zu rücken. Erste Forschungsergebnisse zu einzelnen Teilbereichen weisen darauf hin, dass unsere Heilverfahren sowohl in medizinischer Hinsicht als auch mit Blick auf das zentrale Ziel der Wiedereingliederung der Unfallverletzten in das Erwerbsleben erfolgreich sind und zudem eine günstige Kosten-Nutzen-Relation aufweisen. Um hier weiter gehende Erkenntnisse zu gewinnen, haben wir ein breit angelegtes Forschungsvorhaben auf den Weg gebracht, in dem die Ergebnisse des berufsgenossenschaftlichen Heilverfahrens am Beispiel bestimmter Verletzungsarten evaluiert werden sollen. Über die reine Ergebnisdarstellung hinaus wollen wir das Projekt auch dazu nutzen, Verbesserungspotenzial in den Strukturen und Abläufen der Heilverfahren aufzudecken und die Forschungsergebnisse für die Weiterentwicklung unserer Qualitätssicherung nutzbar machen.

\section{Fazit}

Die vorstehenden Ausführungen verdeutlichen, dass die gesetzliche Unfallversicherung auch abseits gesetzgeberischer Reformen vor großen Herausforderungen und einer Vielzahl von Aufgaben steht. Die Unfallversicherungsträger werden auch in Zeiten eines tief greifenden organisatorischen Wandels nicht in ihrem Bestreben für eine hochwertige und effiziente Rehabilitation nachlassen. Ziel ist es, eine möglichst hohe Behandlungsqualität zu ökonomisch vertretbaren Kosten zu erreichen und nach außen zu dokumentieren.

Die Unfallversicherungsträger werden sich auch in Zukunft neuen Entwicklungen und Herausforderungen stellen und ihre Gestaltungskompetenz weiterhin im Interesse der Versicherten und Beitragszahler zu nutzen wissen.

\section{Korrespondenzadresse}

Dr. J. Breuer

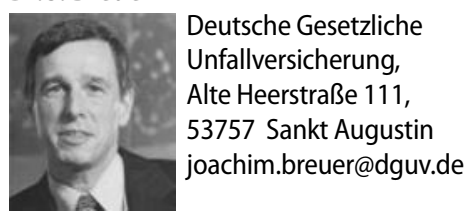

Interessenkonflikt. Keine Angaben 